\title{
Application of the Truncated Zero-Inflated Double Poisson for Determining of the Effecting Factors on the Number of Coronary Artery Stenosis
}

\author{
Arezoo Orooji, ${ }^{1}$ Toktam Sahranavard ${ }^{(D},{ }^{2}$ Mohammad-Taghi Shakeri $\left(\mathbb{D},{ }^{3}\right.$ \\ Mohammad Tajfard, ${ }^{4,5}$ and Seyed Ehsan Saffari ${ }^{6}{ }^{6}$ \\ ${ }^{1}$ Department of Epidemiology and Biostatistics, Faculty of Health Sciences, Mashhad University of Medical Sciences, Mashhad, Iran \\ ${ }^{2}$ Faculty of Medicine, Mashhad University of Medical Science, Mashhad, Iran \\ ${ }^{3}$ Department of Epidemiology \& Biostatistics, MUMS, Mashhad, Iran \\ ${ }^{4}$ Department of Health Education and Health Promotion, Faculty of Health, Mashhad University of Medical Sciences, \\ Mashhad, Iran \\ ${ }^{5}$ Social Determinants of Health Research Center, Mashhad University of Medical Sciences, Mashhad, Iran \\ ${ }^{6}$ Centre for Quantitative Medicine, Duke-NUS Medical School, National University of Singapore, Singapore
}

Correspondence should be addressed to Seyed Ehsan Saffari; ehsan.saffari@duke-nus.edu.sg

Received 27 September 2021; Revised 17 December 2021; Accepted 22 December 2021; Published 13 January 2022

Academic Editor: Kazuhisa Nishizawa

Copyright (C) 2022 Arezoo Orooji et al. This is an open access article distributed under the Creative Commons Attribution License, which permits unrestricted use, distribution, and reproduction in any medium, provided the original work is properly cited.

Background. Risk factors of coronary heart disease have been discussed in the literature; however, conventional statistical models are not appropriate when the outcome of interest is number of vessels with obstructive coronary artery disease. In this paper, a novel statistical model is discussed to investigate the risk factors of number of vessels with obstructive coronary artery disease. Methods. This cross-sectional study was conducted on 633 elderly cardiovascular patients at Ghaem Hospital, Mashhad, Iran from September 2011 to May 2013. Clinical outcome is number of vessels with obstructive coronary artery disease $(=0,1,2,3)$, and predictor variables are baseline demographics and clinical features. A right-truncated zero-inflated double Poisson regression model is performed which can accommodate both underdispersion and excess zeros in the outcome. The goodnessof-fit of the proposed model is compared with conventional regression models. Results. Out of 633 cardiovascular patients, 327 were male $(51.7 \%)$. Mean age was $\sim 65 \pm 7$ years (for individuals with zero, one, and two coronary artery stenosis) and $\sim 66 \pm$ 7 years (for individuals with three coronary artery stenosis). BMI $(0.04 \pm 0.01, p=0.011)$ and female gender $(0.19 \pm 0.09, p=$ $0.032)$ were significant associated with the count part of the model, and only BMI $(-0.47 \pm 0.2, p=0.011)$ was significantly predictive of logit part of the model. The goodness-of-fit measurements indicate that the proposed model outperforms the conventional regression models. Conclusion. The proposal regression model shows a better fit compared to the standard regression analysis in modeling number of vessels with obstructive coronary artery disease. Hence, using truncated zeroinflated double Poisson regression model-as an alternative model-is advised to study the risk factors of number of involved vessels of coronary artery stenosis.

\section{Background}

The cause of one out of three deaths is cardiovascular disease [1]. It is noted that coronary heart disease is one of the two highest causes of mortality in Australia and UK [2]. Coronary heart disease is the most common reason of disability with $13.3 \%$ of deaths worldwide [3]. Coronary artery disease develops when the major blood vessels-that supply heart with blood, oxygen, and nutrients (coronary arteries)-become damaged or narrowed. The narrowing may lead to ischemic heart disease and even death [4]. Coronary angiography is the gold standard diagnostic method for coronary heart disease and an expensive approach in terms of cost and having several side effects [5]. Prognostic factors of 
coronary heart disease have been discussed in the literature, including gender, age, smoking status, diabetes mellitus, systemic hypertension, hypercholesterolemia, high sensitivity $\mathrm{C}$ reactive protein (hs-CRP), and body mass index (BMI) [3, 6-8].

Regression models are commonly used to investigate the association between independent variables and clinical outcome [9]. If the clinical outcome is a count variable, count regression models are recommended. In many clinical data, there is a high proportion of zeros in the outcome-excess zeros. Zero-inflated (ZI) count models are typically used to model such data. In ZI models, a mixture of two separate data generation processes is performed: one generates only zeros, and the other is a count distribution data-generating process. Determining which of the processes leads to observation is done by a Bernoulli experiment. In addition, there are two types of zeros in such data: structural zeros (or true zeros) which refers to a not-at-risk situation for a behavior of interest and random zeros (or false zeros) which refers to an at-risk situation due to sampling variability. The mixture distribution discussed above is one way of accommodating the difference between structural and random zeros [10-12].

In many applications, there are some extreme values at the right side of the distribution which causes overdispersion (variance is greater than mean). One solution to overcome this problem is to truncate the extreme values above a specific point. On the other hand, there are situations where the outcome of interest is right-truncated by definition. An example of this scenario is number of vessels with obstructive coronary artery disease which is limited to a maximum of 3 vessels. If the conventional count regression models (with no truncation) are performed on number of involved vessels with obstructive coronary artery disease, predicted probabilities will be generated for all nonnegative values for number of involved vessels which is not meaningful $[13,14]$. Hence, a righttruncated model seems to be a good approach.

Poisson regression is the most common approach used for modeling count response variables [11, 14]. Mean is assumed to be equal to variance in the Poisson regression model; hence, this model cannot perform well when under and overdispersion scenarios [13]. Overdispersion has been addressed pretty well in literature; however, models which can accommodate underdispersion are limited [11-13]. Number of involved vessels in coronary artery stenosis is an underdispersed outcome in our case study.

The aim of this study is to introduce zero-inflated double Poisson (ZIDP) model with right truncation as an alternative novel approach addressing both excess zeros and underdispersion problems simultaneously for patient with coronary artery stenosis. Validation of this model will be assessed via comparison of the goodness-of-fit measurements (including -2LogLikelihood (-2LL) and Akaike information criterion (AIC)) of the proposed model with other alternative count regression models.

\section{Methods}

2.1. Data Description. This cross-sectional study was conducted on 633 cardiovascular patients who visited cardiovas- cular department and received catheter angiography at Ghaem Hospital, Mashhad, Iran, from September 2011 to May 2013. Inclusion criteria were aged $>55$ years old (as the intention was to target a potentially sicker population) [15-19], and exclusion criteria were pregnant and lactating women and individuals with rheumatic disease, chronic liver disease, kidney disease, any type of cancer, infectious diseases in the last 3 months, history of any surgery in the last 3 months, history of coronary angioplasty, immunological disease, any disease associated with inflammatory bowel disease, and those taking any of the following medications: steroids, penicillin, oral contraceptives, or hormone replacement therapy.

The outcome variable is number of vessels involved in coronary artery stenosis revealed by angiography. Predictor variables included are the known risk factors of coronary artery disease which are available in this study including gender, age, body mass index (BMI), history of smoking, hypertension history, diabetic history, hyperlipidemia history, cardiovascular diseases history, and myocardial infarction history [20, 21].

2.2. Data Analysis and Statistical Methods. The clinical outcome of this study is nonnegative integer value $(=0,1,2,3)$. Demographics and clinical features were reported as mean \pm standard deviation and frequency (percent) for continuous and categorical parameters, respectively, and compared by the number of involved vessels of coronary artery stenosis. Various count regression models were conducted to investigate the association between the number of coronary artery stenosis and the above parameters, including Poisson and negative binomial regression models (as the most commonly used models), zero-inflated Poisson (ZIP) and zeroinflated negative binomial (ZINB) models (due to high proportion of zeros), and right-truncated ZIP and ZINB models (since the number of coronary artery stenosis is $\leq 3$ ). Finally, a right-truncated zero-inflated double Poisson is performed which can accommodate both underdispersion and excess zeros in the outcome. Parameter estimates were obtained using maximum likelihood approach. Goodness-of-fit measures-including -2LogLikelihood and Akaike information criterion-were calculated for each model to assess the validation of the proposed model. Statistical significance was set at $p<0.05$. Statistical analyses were performed using SAS software version 9.4 for Windows (SAS, Inc., Cary, NC).

2.2.1. Double Poisson Regression. Double Poisson regression suggested by Efron (1986) is a count model which can be used for over- and underdispersed data, where variance is greater or less than mean, respectively [22]. This model incorporates a dispersion parameter to handle over- and underdispersion. The probability mass function of a double Poisson distribution is given by

$$
P(Y=y)=f \mu \varphi(y)=c(\mu \varphi)\left(\varphi \frac{1}{2} e^{-\varphi \mu}\right)\left(\frac{e^{-y} y^{y}}{y !}\right)\left(\frac{e \mu}{y}\right)^{j y}, y=0,1,2, \cdots,
$$


where $c(\mu, \varphi)$ is a constant and given by

$$
\frac{1}{c(\mu, \varphi)}=\sum_{y=0}^{\infty} f_{\mu, \varphi}(y) \approx 1+\frac{1-\varphi}{12 \mu \varphi}\left(1+\frac{1}{\mu \varphi}\right),
$$

ensuring the probability mass function is perforce normalized to sum to unity in most cases. $\mu$ and $\phi$ are the mean and dispersion parameters. The link function is defined as $\mu=\exp (x \beta)$, where $x$ is a vector of covariates associated with $Y$, and $\beta$ is a vector of unknown regression coefficients. The expected value and variance of the exact density are approximated by $\mu$ and $\mu / \phi$. Hence, double Poisson allows for both underdispersion $(\phi>1)$ and overdispersion $(\phi<1$ ) . The double Poisson model collapse to Poisson model when $\phi=1$.

2.2.2. Excess Zero with Right Truncation. There are situations where high proportion of zeros is available in count data. Mixture models such as zero-inflated models can handle the excess zeros in the outcome variable. In this study, there is excess zeros in number of coronary artery stenosis. The probability mass function of ZIDP model is given by

$$
p(Y=y)=\left\{\begin{array}{c}
\pi+(1-\pi) c(\mu, \varphi)\left(\varphi^{\frac{1}{2}} e^{-\varphi \mu}\right), y=0 \\
(1-\pi) c(\mu, \varphi)\left(\varphi^{\frac{1}{2}} e^{-\varphi \mu}\right)\left(\frac{e^{-y} y^{y}}{y !}\right)^{\varphi \mathrm{y}}, y>0
\end{array}\right.
$$
$x \beta)$.

where $\pi$ is a link function defined as $\exp (x \beta) / 1+\exp ($

Since the number of coronary artery stenosis in cardiovascular disease cannot be more than three, a righttruncated approach is applied on the ZIDP model. Therefore, probability mass function for truncated zero-inflated double Poisson (TZIDP) is given by

$p(Y=y)=\left\{\begin{array}{l}\frac{\pi+(1-\pi) c(\mu, \varphi)\left(\varphi^{1 / 2} e^{-\varphi \mu}\right)}{1-\sum_{y=4}^{\infty}(1-\pi) c(\mu, \varphi)\left(\varphi^{1 / 2} e^{-\varphi \mu}\right)\left(e^{-y} y^{y} / y !\right)(e \mu / y)^{\varphi y}}, y=0, \\ \frac{(1-\pi) c(\mu, \varphi)\left(\varphi^{1 / 2} e^{-\varphi \mu}\right)\left(e^{-y} y^{y} / y !\right)(e \mu / y)^{\varphi y}}{1-\sum_{y=4}^{\infty}(1-\pi) c(\mu, \varphi)\left(\varphi^{1 / 2} \mathrm{e}^{-\varphi \mu}\right)\left(e^{-y} y^{y} / y !\right)(e \mu / y)^{\varphi y}}, y=1,2,3 .\end{array}\right.$

\section{Results}

In this study, 633 cardiovascular patients participated, of which 327 were male (51.7\%). Mean age was $64.9 \pm 6.68$, $65.0 \pm 6.51,64.9 \pm 6.71$, and $66.4 \pm 7.12$ years for individuals with zero, one, two, and three coronary artery stenosis, respectively. Patients' BMI with zero, one, two, and three coronary artery stenosis was $26.7 \pm 5.4,26.6 \pm 4.47,27.5 \pm$ 6.18 , and $26.7 \pm 5.47$, respectively (Table 1 ). Baseline clinical features of patients-high sensitivity $\mathrm{C}$ reaction, smoking status, hypertension, diabetes, hyperlipidemia, history of cardiovascular diseases, and history of myocardial infarctio$\mathrm{n}$-are reported by number of coronary artery stenosis in Table 1.
Truncated zero-inflated double Poisson model was performed on the number of coronary artery stenosis outcome. BMI and sex were significant predictors of the outcome in the count part of the model. Higher BMI $(0.04 ; 95 \% \mathrm{CI}$ : (0.01, 0.04), $p=0.011)$ and being female $(0.19 ; 95 \% \mathrm{CI}$ : $(0.02,0.36), p=0.032)$ are found to be associated with higher number of coronary artery stenosis.

In regards to the results of logit part, BMI was the only significant factor. For every one-unit increase in BMI, the odds that the number of coronary artery stenosis would be a certain zero decrease by a factor of $\exp (-0.47)=0.63$ $(p=0.011)$. In other words, it is less likely that the number of coronary artery stenosis is zero as BMI increases.

Dispersion of the fitted outcome variable was investigated in the TZIDP model. The estimate of dispersion parameter $(\phi)$ was $0.23(p<0.001)$, suggesting that the estimated mean is less than the estimated variance in number of coronary artery stenosis outcome (Table 2).

To assess the validation of TZIDP model, goodness-offit statistics (including -2LogLikelihood and Akaike information criterion) were calculated and compared with alternative count models. The TZIDP model has shown the lowest goodness-of-fit statistics among all models (Table 3).

\section{Discussion}

In this study, we have established for the first time the truncated zero-inflated double Poisson model to characterize the effecting factors of the number of coronary artery stenosis.

In the present study, TZIDP, ZIDP, ZIP, ZINB, NB, and Poisson models were considered, and their performance was evaluated via goodness-of-fit measurements. The -2LL and AIC statistics indicate that TZIDP outperformed the other candidate models.

The descriptive results of the outcome variables show that the mean value $(=1.7)$ is greater than the variance $(=$ 1.5), indicating underdispersion in the raw data. Although the difference $(=0.2=1.7-1.5)$ is not significant, the double Poisson model was conducted. Once the TZIDP model is fit, the estimation of the dispersion parameter is in favor of overdispersion $(\phi=0.23<1)$, suggesting that the estimated mean is less than the estimated variance in the fitted number of coronary artery stenosis. The advantage of using double Poisson model can be noted in this case, where there is no clear and strong evidence of under- or overdispersion in the observed data. The underlying assumption of traditional statistical distributions such as Poisson (equidispersion assumption) and negative binomial (over-dispersion assumption) models may not be tenable on the observed data before conducting the models. Under extreme cases, where the underdispersion scenario is obvious in the raw data, the above models may even encounter some convergence issues. Double Poisson model, on the other hand, has a dispersion parameter which makes the distribution more flexible to handle both over- and underdispersion scenarios. When designing the statistical analysis plan for a prospective study, it is not uncommon to have limited information about the expected distribution of a count variable 
TABLe 1: Demographic variables and baseline clinical features.

\begin{tabular}{|c|c|c|c|c|}
\hline \multirow[b]{2}{*}{ Variable } & \multicolumn{4}{|c|}{ Number of coronary artery stenosis* } \\
\hline & $\begin{array}{c}0 \\
(n=157)\end{array}$ & $\begin{array}{c}1 \\
(n=104)\end{array}$ & $\begin{array}{c}2 \\
(n=134)\end{array}$ & $\begin{array}{c}3 \\
(n=238)\end{array}$ \\
\hline Age & $64.9 \pm 6.68$ & $65.0 \pm 6.51$ & $64.9 \pm 6.71$ & $66.4 \pm 7.12$ \\
\hline Body mass index & $26.7 \pm 5.4$ & $26.6 \pm 4.47$ & $27.5 \pm 6.18$ & $26.7 \pm 5.47$ \\
\hline High sensitivity $\mathrm{C}$ reactive protein & $5.0 \pm 5.36$ & $7.0 \pm 1.00$ & $7.1 \pm 9.03$ & $6.8 \pm 7.53$ \\
\hline Gender (female) & $109(69.4)$ & $46(44.2)$ & $60(44.8)$ & $91(38.2)$ \\
\hline History of smoking & $44(28.0)$ & $41(39.4)$ & $47(35.1)$ & $83(34.9)$ \\
\hline Hypertension history & $80(51.0)$ & $50(48.1)$ & $63(47.0)$ & $126(52.9)$ \\
\hline Diabetic history & $43(27.4)$ & $38(36.9)$ & $44(33.1)$ & $97(40.9)$ \\
\hline Hyperlipidemia history & $56(35.7)$ & $40(38.5)$ & $54(40.3)$ & $95(39.9)$ \\
\hline Cardiovascular diseases history & $78(49.7)$ & $49(47.1)$ & $48(35.8)$ & $102(42.9)$ \\
\hline Myocardial infarction history & $10(6.8)$ & $16(16.5)$ & $26(20.6)$ & $46(20.3)$ \\
\hline
\end{tabular}

${ }^{*}$ Mean \pm standard deviation for continuous variables; frequency (\%) for categorical variables.

TABle 2: Parameter estimates using truncated zero-inflated double Poisson.

\begin{tabular}{lccc}
\hline Parameter & Estimate & $95 \%$ confidence interval & $p$ value \\
\hline Count part & & & \\
Body mass index & 0.037 & $(0.01,0.06)$ & 0.011 \\
Age (year) & -0.0005 & $(-0.017,0.016)$ & 0.956 \\
Hypertension history & -0.047 & $(-0.497,0.4)$ & 0.837 \\
Diabetic history & 0.041 & $(-0.739,0.821)$ & 0.916 \\
Gender (female) & 0.19 & $(0.02,0.36)$ & 0.032 \\
Myocardial infarction history & 0.15 & $(-0.08,0.38)$ & 0.228 \\
High sensitivity C reactive protein & 0.011 & $(-0.001,0.023)$ & \\
Logit part & & & 0.086 \\
Body mass index & -0.47 & $(-0.82,-0.12)$ & $(-0.20,0.26)$ \\
Age (year) & 0.03 & $(-4.90,6.04)$ & 0.011 \\
Hypertension history & 0.57 & $(-9.71,10.95)$ & 0.801 \\
Diabetic history & 0.62 & $(0.09,0.37)$ & 0.837 \\
Dispersion $(\phi)$ & 0.23 & & 0.905 \\
\hline
\end{tabular}

TABLE 3: Goodness-of-fit measurements of regression models.

\begin{tabular}{lcc}
\hline \multirow{2}{*}{ Model } & \multicolumn{2}{c}{$\begin{array}{c}\text { Goodness-of-fit } \\
\text { statistics }\end{array}$} \\
& -2LL & AIC \\
\hline Truncated zero-inflated double Poisson & 1522.4 & 1550.4 \\
Zero-inflated double Poisson & 1734.8 & 1762.8 \\
Zero-inflated Poisson regression & 1850.2 & 1876 \\
Zero-inflated negative binomial regression & 1850.2 & 1876 \\
Negative binomial regression & 1865.7 & 1882 \\
Poisson regression & 1865.7 & 1882 \\
\hline
\end{tabular}

AIC: Akaike Information Criterion; -2LL: -2LogLikelihood.

whether over- or underdispersed. Double Poisson could be a smart choice in such situation which can offer flexibility around the dispersion parameter. Nevertheless, the nonlinear link function (mean of TZINB model is a nonlinear function of the model parameters) and the nonlinear rela- tionship between dispersion parameter and mean and variance parameters could also explain the above result [23-25].

This literature shows that the zero-inflated models perform a better fit in the presence of excess zeros in the outcome. Using similar approach, various models have been discussed in this study to investigate the performance of conventional count models (ZIP vs. Poisson; ZINB vs. NB). In the presence of excess zeros, zero-inflated models are commonly used in literature. In this study, the goodnessof-fit measurements of ZI models are significantly lower than the non-ZI corresponding models, which is an expected finding. Zero-inflated regression models, by considering different probability functions for zeros and positive counts, would result in a less biased predicted values in compared to the observed values for the outcome. This has been also shown in literature $[10,13,14]$.

The right truncation method is applied in this study as the outcome variable cannot take values $>3$ due to theoretical range being $0,1,2$, and 3 . Without truncation, there will 
be predicted probabilities for the values out of the meaningful clinical range. This is a critical point which should not be ignored. Also, there is a significant improvement in the performance fit once right truncation model is applied. This is an expected result as the probabilities will be limited to the truncated range of the outcome values, which makes the predicted values to be closer to the observed values, and no prediction for the values out of the theoretical range will be calculated. Such improvement in the model fit with right truncation is also shown in literature [13, 26, 27].

In this study, the TZIDP model outperformed the alternative models based on the goodness-of-fit statistics, dealing with the excess zeros and right-truncated data with no underlying assumption about the outcome dispersion, i.e., TZIDP can handle dual data anomalies of excess zeros and right-truncated data in a count response variable where there is not a clear evidence of under- or overdispersion. Similar methodological ideas have been also reported in literature where there was some initial signals of overdispersion in the raw data [27-29].

It should be also noted that TZIDP is a complex model with many parameters. As a result, interpretations should be made with caution, and the estimates of the "count part" and the "logit part" of the model should be consistent for the same variable. For example, higher BMI in Table 3 is shown to be associated with higher number of coronary artery stenosis (count part) and lower likelihood of coronary artery stenosis being zero (logit part), which is consistent. Furthermore, the proposed model is able to improve the model fit significantly (Table 2); hence, it would be preferable in compared to the alternative models, aside from model complexity.

According to the fact that the number of coronary artery stenosis is zero in most patients, the proposed statistical can be an alternative approach to addressee both excess zeros and dispersion problems simultaneously for patients with coronary artery stenosis. The most important clinically relevant finding was that BMI and sex were significant predictors of the number of coronary artery stenosis in count part of the truncated zero-inflated double Poisson model, and BMI was the only significant predictor in logit part of the model.

Based on our findings, BMI is associated with the number of involved vessels which is consistent with some previous studies results. Indeed, our research confirmed other observations which considered BMI as an independent predictor in coronary endothelial dysfunction in patients with coronary artery disease [30, 31]. However, this finding is contrary to number of previous studies which have suggested that BMI was not an independent factor of coronary artery disease or severity on multivariate stepwise linear regression analysis anymore [32, 33]. This inconsistency might be due to the fact that our cohort is biased towards sever cardiovascular cases as well as elderly patients.

Several investigations have been previously reported gender differences in the distribution of risk factors of the number of coronary artery stenosis. In spite of differences between researches, our findings are consistent with previous reports $[34,35]$. Our study showed that the number of coronary artery stenosis in women was 1.2 times more than men $(p=0.032)$. Due to the misperception of the protection against cardiovascular disease in females, the risk of heart disease is often underestimated in female group. As a result, aggressive treatment is less common on women rather than men in clinical trials, while the results of some studies have demonstrated that the number of coronary arteries involved was higher in men than women [36]. This result may be explained by the principle that the risk of coronary artery stenosis increases with age. Due to the effect of sex hormone function, women before menopause age are protected against coronary artery disease [37]. However, our results did not presume that age has a direct impact on the number of involved coronary arteries. Such contradiction in the impact of age could be due to the inclusion criteria of the current study where the intention was on elderly patients (>55 years old).

This study did not find a significant correlation between hs-CRP and coronary artery stenosis. Similar results did not find any association between CRP levels and angiographic extent score $[38,39]$. These results reflect the finding of a review which evaluated the prognostic value of CRP in coronary artery disease. It was concluded that there were different biases in 83 previous studies, and the relationship between hs-CRP and prognosis is not strong [40].

Our outcome showed no significant correlation between diabetes mellitus and number of involved vessels (according to the classification in one, two and three vessel), but there was a probable considerable difference if it was evaluated based on one vessel and more than one vessel [33]. Other studies have been established that diabetics are more prone to multivessel coronary disease [41].

Concerning the amount of blood pressure, no statistically significant relationship between hypertension and the number of involved vessels was asserted. A positive relationship was reported in many studies $[42,43]$. In spite of proved relation among hypertension, atherosclerosis, and coronary artery disease, other studies documented no significant association between hypertension and number of involved vessels. It should not be ignored that the focus of the current study is on severe cardiovascular elderly patients which might result in slightly different conclusion from the literature.

Angiography only shows lumen vessels and not the cross-sectional vessel wall morphology of vessel. Myocardial infarction usually is a consequence of angiographically nonobstructive lesions. This reality can explain why our results showed no significant relation between history of myocardial infarction and number of involved vessels [43].

\section{Conclusion}

The strength of this study was the novel methodology. As most patients had no coronary artery stenosis and number of involved vessels is limited to $\leq 3$, the proposed model outperformed the conventional statistical methods. Potential limitation of the current study is the small sample size (single center study over $<2$ years of data collection) and the study design in terms of the eligibility criteria which limits the study population to severe cardiovascular elderly 
patients. Further investigations are required in larger studies, and the results could be validated in external cohorts of patients. Using the TZIDP model-as an alternative mode1 -is advised to study the risk factors of number of involved vessels of coronary artery stenosis.

\section{Data Availability}

All data and materials are available from the corresponding author upon reasonable request.

\section{Ethical Approval}

All participants volunteered and received a data protection declaration in agreement with the Helsinki Declaration. They gave both, written and verbal, informed consent. The study was approved according to the ethical guidelines by the Ethical commission of the Mashhad University of Medical Science (EK 900671).

\section{Consent}

This study has patient consent to the publication of deidentified information.

\section{Conflicts of Interest}

The authors declare that they have no competing interests.

\section{Authors' Contributions}

AO drafted the paper and performed the data analysis. TS, MTS, MT, and SES provided comments on the interpretation of the results and contributed equally and significantly in revising the results and discussion of the manuscript. AO and SES designed the study and worked on the methodology. All authors read and approved the final manuscript.

\section{References}

[1] H. Shah, M. U. Jan, A. Altaf, and M. Salahudin, "Correlation of hyper-homocysteinemia with coronary artery disease in absence of conventional risk factors among young adults," Journal of the Saudi Heart Association, vol. 30, no. 4, pp. 305-310, 2018.

[2] J. Nahar, T. Imam, and K. S. Tickle, "Association rule mining to detect factors which contribute to heart disease in males and females," Expert Systems with Applications, vol. 40, no. 4, pp. 1086-1093, 2013.

[3] M. Tayefi, M. Tajfard, S. Saffar et al., "Hs-CRP is strongly associated with coronary heart disease (CHD): a data mining approach using decision tree algorithm," Computer Methods and Programs in Biomedicine, vol. 141, pp. 105-109, 2017.

[4] P. Libby, P. M. Ridker, and G. K. Hansson, "Inflammation in atherosclerosis: from pathophysiology to practice," Journal of the American College of Cardiology, vol. 54, no. 23, pp. 21292138, 2009.

[5] R. Alizadehsani, J. Habibi, M. J. Hosseini et al., "A data mining approach for diagnosis of coronary artery disease," Computer Methods and Programs in Biomedicine, vol. 111, no. 1, pp. 52-61, 2013.
[6] I. Kurt, M. Ture, and A. T. Kurum, "Comparing performances of logistic regression, classification and regression tree, and neural networks for predicting coronary artery disease," Expert Systems with Applications, vol. 34, no. 1, pp. 366-374, 2008.

[7] J. Soni, U. Ansari, D. Sharma, and S. Soni, "Predictive data mining for medical diagnosis: an overview of heart disease prediction," International Journal of Computer Applications, vol. 17, no. 8, pp. 43-48, 2011.

[8] I. Nikbakht-Jam, H. Mohaddes-Ardabili, P. Keshavarz et al., "A comparison of obesity and some cardiovascular risk factors between male employees of gas refinery, petrochemical plant, and non-industrial workplaces," Iranian journal of health sciences, vol. 6, no. 1, pp. 1-8, 2018.

[9] J. F. Lawless and Y. Yuan, "Estimation of prediction error for survival models," Statistics in Medicine, vol. 29, no. 2, pp. 262-274, 2010.

[10] S. Yang, A Comparison of Different Methods of Zero-Inflated Data Analysis and Its Application in Health Surveys, University of Rhode Island, 2014.

[11] D. Erdman, L. Jackson, and A. Sinko, "Zero-inflated Poisson and zero-inflated negative binomial models using the COUNTREG procedure," SAS Global Forum, vol. 2008, pp. 322-2008, 2008.

[12] Y. B. Cheung, "Zero-inflated models for regression analysis of count data: a study of growth and development," Statistics in Medicine, vol. 21, no. 10, pp. 1461-1469, 2002.

[13] S. E. Saffari, R. Adnan, and W. Greene, "Handling of overdispersion of count data via truncation using poisson regression model," Journal of Computer Science and Computational Mathematics, vol. 1, no. 1, pp. 1-4, 2011.

[14] S. E. Saffari, R. Adnan, W. Greene, and M. H. Ahmad, "A Poisson regression model for analysis of censored count data with excess zeroes," Jurnal Teknologi, vol. 63, no. 2, 2013.

[15] J. J. Díez, "Hyperthyroidism in patients older than 55 years: an analysis of the etiology and management," Gerontology, vol. 49, no. 5, pp. 316-323, 2003.

[16] S. Aw, G. Koh, Y. J. Oh et al., "Explaining the continuum of social participation among older adults in Singapore: from 'closed doors' to active ageing in multi-ethnic community settings," Journal of Aging Studies, vol. 42, pp. 46-55, 2017.

[17] Y. Lu, C. Liu, D. Yu et al., "Prevalence of mild cognitive impairment in community-dwelling Chinese populations aged over 55 years: a meta-analysis and systematic review," BMC Geriatrics, vol. 21, no. 1, pp. 1-16, 2021.

[18] R. Hu, S. Zhao, D. Wang et al., "A prevalence study on mild cognitive impairment among the elderly populations of Mongolian and Han nationalities in a pastoral area of Inner Mongolia," Zhonghua liu xing bing xue za zhi= Zhonghua liuxingbingxue zazhi, vol. 33, no. 4, pp. 364-367, 2012.

[19] M. Lao, H. Zhang, G. Luo, X. Yi, Y. Huang, and Z. Wu, "Prevalence of mild cognitive impairment amony 55-years old or over individuals in Hainan Island," Hainan Medical Journal (Hai Nan Yi Xue), vol. 22, no. 14, pp. 112-114, 2011.

[20] Y. Si, W. Fan, W. Shan et al., "Association between triglyceride glucose index and coronary artery disease with type 2 diabetes mellitus in middle-aged and elderly people," Medicine, vol. 100, no. 9, p. e25025, 2021.

[21] N. Suppogu, J. Wei, O. Quesada et al., "Angina relates to coronary flow in women with ischemia and no obstructive coronary artery disease," International Journal of Cardiology, vol. 333, pp. 35-39, 2021. 
[22] B. Efron, "Double exponential families and their use in generalized linear regression," Journal of the American Statistical Association, vol. 81, no. 395, pp. 709-721, 1986.

[23] S. Gurmu, "Generalized hurdle count data regression models," Economics Letters, vol. 58, no. 3, pp. 263-268, 1998.

[24] F.-c. Xie, J.-g. Lin, and B.-c. Wei, "Score tests for zero-inflated double poisson regression models," Acta Math. Appl. Sin. Engl. Ser., vol. 33, no. 4, pp. 851-864, 2017.

[25] Y. Zou, S. R. Geedipally, and D. Lord, "Evaluating the double Poisson generalized linear model," Accident Analysis \& Prevention, vol. 59, pp. 497-505, 2013.

[26] S. E. Saffari and R. Adnan, "Parameter estimation on zeroinflated negative binomial regression with right truncated data," Sains Malaysiana, vol. 41, pp. 1483-1487, 2012.

[27] S. E. Saffari, J. C. Allen, R. Adnan, S. H. Ong, S. Z. Sim, and W. Greene, "Frequency of visiting a doctor: a right truncated count regression model with excess zeros," Biostatistics and Biometrics Open Access Journal, vol. 9, no. 5, pp. 112-122, 2019.

[28] S. E. Saffari, R. Adnan, and W. Greene, "Hurdle negative binomial regression model with right censored count data. Sortstatistics and operations research," Transactions, pp. 181194, 2012.

[29] S. E. Saffari, R. Adnan, and W. Greene, "Investigating the impact of excess zeros on hurdle-generalized Poisson regression model with right censored count data," Statistica Neerlandica, vol. 67, no. 1, pp. 67-80, 2013.

[30] K. Pourmand, M. Sadeghi, H. SANEEI, F. Akrami, and M. Talaei, Which MAJOT ATHEROSCLEROSIS'RISK Factors Represents the Extent of Coronary Artery Disease?, 2007.

[31] A. J. Flint, K. M. Rexrode, F. B. Hu et al., "Body mass index, waist circumference, and risk of coronary heart disease: a prospective study among men and women," Obesity Research \& Clinical Practice, vol. 4, no. 3, pp. e171-e181, 2010.

[32] A. Niraj, J. Pradahan, H. Fakhry, V. Veeranna, and L. Afonso, "Severity of coronary artery disease in obese patients undergoing coronary angiography: "obesity paradox" revisited," Clinical Cardiology, vol. 30, no. 8, pp. 391-396, 2007.

[33] A. Golmohammadi, M. T. Sadeghi, M. Bakhshayeshi, H. Namdar, and A. Separham, "Relation of atherosclerosis risk factors with the number of involved coronary arteries in angiography," Medical Science and Discovery, vol. 3, no. 2, pp. 6570, 2016.

[34] D. Hochner-Celnikier, O. Manor, O. Gotzman, H. Lotan, and T. Chajek-Shaul, "Gender gap in coronary artery disease: comparison of the extent, severity and risk factors in men and women aged 45-65 years," Cardiology, vol. 97, no. 1, pp. 1823, 2002 .

[35] V. Vaccarino, Ischemic Heart Disease in Women: Many Questions, Few Facts, Am Heart Assoc, 2010.

[36] A. J. Lansky, V. G. Ng, A. Maehara et al., "Gender and the extent of coronary atherosclerosis, plaque composition, and clinical outcomes in acute coronary syndromes," JACC: Cardiovascular Imaging, vol. 5, no. 3, pp. S62-S72, 2012.

[37] S. M. B. Tabei, S. Senemar, B. Saffari, Z. Ahmadi, and S. Haqparast, "Non-modifiable factors of coronary artery stenosis in late onset patients with coronary artery disease in southern Iranian population," Journal of cardiovascular and thoracic research, vol. 6, no. 1, pp. 51-55, 2014.

[38] J. Auer, M. Rammer, R. Berent, T. Weber, E. Lassnig, and B. Eber, "Relation of C-reactive protein levels to presence, extent, and severity of angiographic coronary artery disease," Indian Heart Journal, vol. 54, no. 3, pp. 284-288, 2002.

[39] T. Nyandak, A. Gogna, S. Bansal, and M. Deb, "High sensitive C-reactive protein (hs-CRP) and its correlation with angiographic severity of coronary artery disease (CAD)," J Indian Acad Clin Med, vol. 8, no. 3, pp. 217-221, 2007.

[40] D. Silva and A. P. de Lacerda, "Proteina C reativa de alta sensibilidade como biomarcador de risco na doença coronaria," Revista Portuguesa de Cardiologia (English Edition), vol. 31, no. 11, pp. 733-745, 2012.

[41] S. Uddin, F. Malik, M. Bari et al., "Angiographic severity and extent of coronary artery disease in patients with type 2 diabetes mellitus," Mymensingh medical journal: MMJ, vol. 14, no. 1, pp. 32-37, 2005.

[42] Y. Guo, W. Zhang, Y. Zhou, D. Zhao, Z. Zhou, and H. Zhang, "Study of the relationship between cardiovascular risk factors and severity of coronary artery disease in patients underwent coronary angiography," Zhonghua Xin Xue Guan Bing Za Zhi, vol. 33, no. 5, pp. 415-418, 2005.

[43] V. Veeranna, J. Pradhan, A. Niraj, H. Fakhry, and L. Afonso, "Traditional cardiovascular risk factors and severity of angiographic coronary artery disease in the elderly," Preventive Cardiology, vol. 13, no. 3, pp. 135-140, 2010. 